\title{
Controlled vapour-phase deposition synthesis and growth mechanism of $\mathrm{Bi}_{2} \mathrm{Te}_{3}$
}

nanostructures

W. Lei' , I. Madni, Y. L. Ren, C. L. Yuan, G. Q. Luo, and L. Faraone

Citation: Appl. Phys. Lett. 109, 083106 (2016); doi: 10.1063/1.4961632

View online: http://dx.doi.org/10.1063/1.4961632

View Table of Contents: http://aip.scitation.org/toc/apl/109/8

Published by the American Institute of Physics

Articles you may be interested in

Two-dimensional nanoplates of $\mathrm{Bi} 2 \mathrm{Te} 3$ and $\mathrm{Bi} 2 \mathrm{Se} 3$ with reduced thermal stability

Appl. Phys. Lett. 6, 025110025110 (2016); 10.1063/1.4942113

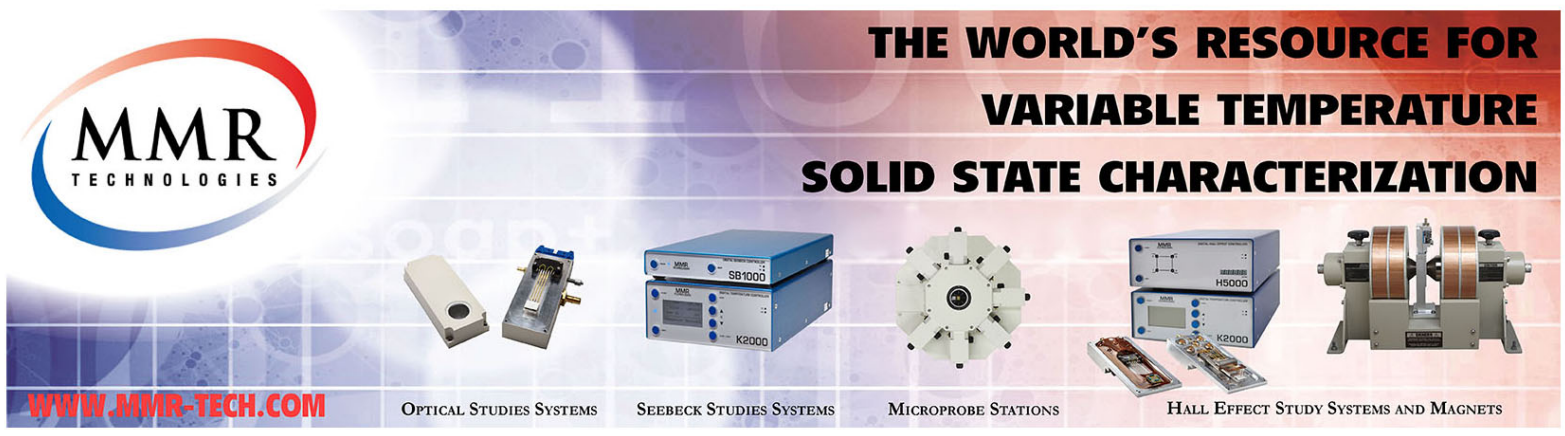




\title{
Controlled vapour-phase deposition synthesis and growth mechanism of $\mathrm{Bi}_{2} \mathrm{Te}_{3}$ nanostructures
}

\author{
W. Lei, ${ }^{1}{ }^{1, a)}$ I. Madni, ${ }^{1}$ Y. L. Ren, ${ }^{1}$ C. L. Yuan, ${ }^{2}$ G. Q. Luo, ${ }^{3}$ and L. Faraone ${ }^{1}$ \\ ${ }^{1}$ School of Electrical, Electronic and Computer Engineering, The University of Western Australia, \\ 35 Stirling Highway, Crawley 6009, Australia \\ ${ }^{2}$ Department of Physics, Jiangxi Normal University, Nanchang, 330022 Jiangxi, People's Republic of China \\ ${ }^{3}$ School of Electronics and Information, Hangzhou Dianzi University, Xiasha High Education Park, \\ Hangzhou 310018, People's Republic of China
}

(Received 17 May 2016; accepted 13 August 2016; published online 26 August 2016)

\begin{abstract}
This work presents a study on the controlled growth and the growth mechanism of vapour-phase deposited two-dimensional $\mathrm{Bi}_{2} \mathrm{Te}_{3}$ nanostructures by investigating the influence of growth conditions on the morphology of $\mathrm{Bi}_{2} \mathrm{Te}_{3}$ nanostructures. The formation of a hexagonal plate geometry for $\mathrm{Bi}_{2} \mathrm{Te}_{3}$ nanostructures is a consequence of the large difference in growth rate between crystal facets along $\langle 0001\rangle$ and $\langle 11 \overline{2} 0\rangle$ directions. Under low Ar carrier gas flow rates $(60-100 \mathrm{sccm})$, the growth of $\mathrm{Bi}_{2} \mathrm{Te}_{3}$ nanoplates occurs in the mass-transport limited regime, whereas under high carrier gas flow rates $(130 \mathrm{sccm})$, the growth of $\mathrm{Bi}_{2} \mathrm{Te}_{3}$ nanoplates is in the surface-reaction limited regime. This leads to an increase in the lateral size of $\mathrm{Bi}_{2} \mathrm{Te}_{3}$ nanoplates with increasing the Ar carrier gas flow rate from 60 to $100 \mathrm{sccm}$, and a decrease in size for a flow rate of $130 \mathrm{sccm}$. In addition, the lateral size of $\mathrm{Bi}_{2} \mathrm{Te}_{3}$ nanoplates was found to increase with increasing growth time due to the kinetic characteristics of material growth. The proposed growth model provides an effective guide for achieving controlled growth of $\mathrm{Bi}_{2} \mathrm{Te}_{3}$ nanoplates, as well as other two dimensional nanomaterials. Published by AIP Publishing. [http://dx.doi.org/10.1063/1.4961632]
\end{abstract}

Recently, significant attention has been devoted to bismuth telluride $\left(\mathrm{Bi}_{2} \mathrm{Te}_{3}\right)$ semiconductor materials due to its new applications in three-dimensional (3D) topological insulator (TI) ${ }^{1,2}$ This new state of quantum matter has an insulating bulk bandgap combined with gapless edges that have current-carrying massless Dirac surface states, ${ }^{1,2}$ which provides a platform for exploring new fundamental physics with potential applications in spintronics, quantum information, and low-energy dissipation electronics. Thus, the material synthesis of this family of compounds is an attractive and necessary precursor for the study of TIs. Compared with bulk materials, low-dimensional TI structures, such as one dimensional (1D) nanowires and two dimensional (2D) nano-sheets, are more attractive for exploring TI surface states due to their large surface-to-volume ratio, thus favouring the manifestation of surface states during transport measurements. Regarding the synthesis/growth of lowdimensional $\mathrm{Bi}_{2} \mathrm{Te}_{3}$ materials, three main methods have been reported: (1) mechanical exfoliation of thin sheets from bulk crystals, ${ }^{3}$ which can achieve thin $\mathrm{Bi}_{2} \mathrm{Te}_{3}$ layers, but with very low yield and irregular shapes, which is not preferred; (2) thin film growth via molecular beam epitaxy (MBE) or Metal Organic Chemical Vapor Phase Deposition (MOCVD), ${ }^{4,5}$ which can also obtain high quality thin film $\mathrm{Bi}_{2} \mathrm{Te}_{3}$ materials, but is relatively expensive and less accessible; and (3) conventional low pressure chemical-vapourdeposition (CVD) processes based on vapour-solid or vapour-liquid-solid growth mechanisms. ${ }^{6,7}$ Because of the low cost and simple operation, conventional CVD methods have been widely applied to the growth of high quality low dimensional materials, including $\mathrm{Bi}_{2} \mathrm{Te}_{3}$ nanostructures.

\footnotetext{
${ }^{\text {a)} E l e c t r o n i c ~ m a i l: ~ w e n . l e i @ u w a . e d u . a u ~}$
}

Over the past several years, $\mathrm{Bi}_{2} \mathrm{Te}_{3}$ nanostructures have been synthesized and studied. ${ }^{7-13}$ However, most of these studies have focussed on fundamental TI properties, and the growth mechanism of these $\mathrm{Bi}_{2} \mathrm{Te}_{3}$ nanostructures is not well understood. For example, there is very little reported in the literature on how to achieve controlled growth of these $\mathrm{Bi}_{2} \mathrm{Te}_{3}$ nanostructures and the related growth mechanism. However, for eventual device applications as well as for high quality fundamental physics study, it is essential to have a better understanding of the growth mechanism in order to achieve controlled growth of $\mathrm{Bi}_{2} \mathrm{Te}_{3}$ nanostructures. In this work, we present a study on the controlled CVD growth of 2D $\mathrm{Bi}_{2} \mathrm{Te}_{3}$ nano-sheets, and the associated growth mechanism. $\mathrm{Bi}_{2} \mathrm{Te}_{3}$ nano-sheets were obtained via vapour phase deposition, and various growth conditions were studied in order to obtain $\mathrm{Bi}_{2} \mathrm{Te}_{3}$ nano-sheets of different lateral dimensions. The associated growth mechanism of these 2D nanosheets is also discussed, which provides an effective pathway towards achieving controlled growth of $\mathrm{Bi}_{2} \mathrm{Te}_{3}$ nano-sheets.

Growth of $\mathrm{Bi}_{2} \mathrm{Te}_{3}$ nanostructures was carried out in a two zone horizontal tube furnace (MTI OTF-1200X-II) via a catalyst-free vapor transport and deposition process. A powdered form of $\mathrm{Bi}_{2} \mathrm{Te}_{3}$ (99.99\% $\mathrm{Bi}_{2} \mathrm{Te}_{3}$, Sigma-Aldrich) was used as the precursor for evaporation, while oxidized silicon wafers were used as the growth substrate and placed downstream at certain positions in order to accurately set the deposition temperature. During growth of the $\mathrm{Bi}_{2} \mathrm{Te}_{3}$ nanostructrues, the $\mathrm{Bi}_{2} \mathrm{Te}_{3}$ precursor was kept at $480^{\circ} \mathrm{C}$, while the $\mathrm{SiO}_{2} / \mathrm{Si}$ substrate was kept at $325^{\circ} \mathrm{C}$. Ultra-pure argon (Ar) gas was used as the carrier gas to transport the precursor vapour downstream onto the substrate surface. To investigate the influence of growth conditions on the morphology of $\mathrm{Bi}_{2} \mathrm{Te}_{3}$ nanostructures, the $\mathrm{Ar}$ carrier gas flow rate was 
varied from $60 \mathrm{sccm}$ (standard cubic centimeters per minute) to $130 \mathrm{sccm}$, while the growth time was varied from the $10 \mathrm{~min}$ to $2 \mathrm{~h}$. The surface morphology of the $\mathrm{Bi}_{2} \mathrm{Te}_{3}$ nanostructures was characterized with scanning electron microscopy (SEM, Hitachi TM3030) and atomic force microscopy (AFM, Witec alpha 300RA ${ }^{+}$). The chemical composition of the $\mathrm{Bi}_{2} \mathrm{Te}_{3}$ nanostructures was analysed with micro-Raman spectroscopy (Renishaw inVia). The crystalline structure of the $\mathrm{Bi}_{2} \mathrm{Te}_{3}$ nanostructures was characterized with transmission electron microscopy (TEM, FEI Titan G2 80-200 TEM/ STEM).

Figure 1(a) shows a representative SEM image of the $\mathrm{Bi}_{2} \mathrm{Te}_{3}$ nanostructures grown under the conditions of 100 sccm Ar carrier gas flow and $1 \mathrm{~h}$ growth time, labelled as sample $\mathrm{A}$. It is observed that $2 \mathrm{D}$ hexagonal $\mathrm{Bi}_{2} \mathrm{Te}_{3}$ nanoplates are formed on the sample surface, which typically present an average lateral dimension of $14 \mu \mathrm{m}$. Figure 1(b) presents the AFM image and the thickness line profile of a representative $\mathrm{Bi}_{2} \mathrm{Te}_{3}$ nanoplate. On average, these nanoplates present a height/thickness of $\sim 11 \mathrm{~nm}$, corresponding to $\sim 11$ quintuple layer (QL) $\mathrm{Bi}_{2} \mathrm{Te}_{3}$. ${ }^{7}$ Thus, the ratio of lateral to vertical dimension of these nanoplates is $>1270$, indicating a highly anisotropic growth, the reason for which will be discussed later in this work. As indicated by the line profile in Figure 1(b), the nanoplate surface is extremely flat on the nm scale, indicating highly uniform growth of these nanoplates. Figure 1(c) shows the Raman spectrum of a representative $\mathrm{Bi}_{2} \mathrm{~T}_{3}$ nanoplate. The four Raman peaks observed
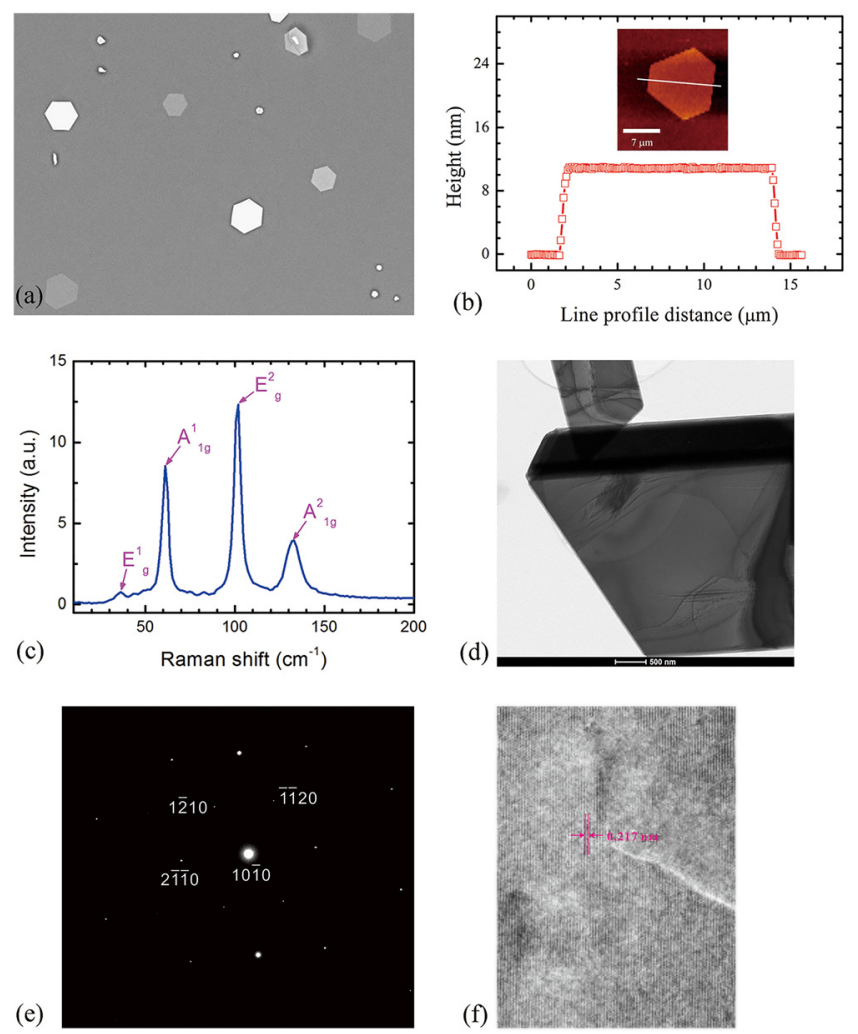

FIG. 1. Representative (a) SEM image, (b) AFM thickness line profile, (c) Raman spectrum, (d) low magnification TEM image, (e) SEAD pattern, and (f) high resolution TEM lattice fringes of $\mathrm{Bi}_{2} \mathrm{Te}_{3}$ nanoplates grown on sample A. The inset of (b) shows the AFM image of the $\mathrm{Bi}_{2} \mathrm{Te}_{3}$ nanoplate measured, where the white line marks the line profile location. in Figure 1(c) are located at 36.3, 61.1, 101.5, and $132.6 \mathrm{~cm}^{-1}$, corresponding to the $\mathrm{E}_{\mathrm{g}}^{1}, \mathrm{~A}^{1}{ }_{1 \mathrm{~g}}, \mathrm{E}_{\mathrm{g}}^{2}$, and $\mathrm{A}^{2}{ }_{1 \mathrm{~g}}$ vibration modes of $\mathrm{Bi}_{2} \mathrm{Te}_{3}$, respectively, and thus confirming the growth of $\mathrm{Bi}_{2} \mathrm{Te}_{3}$ material. ${ }^{14}$ Figures $1(\mathrm{~d})-1(\mathrm{f})$ shows a low magnification TEM image, the spot pattern of selected area electron diffraction (SAED), and the corresponding high-resolution TEM lattice fringes of a representative $\mathrm{Bi}_{2} \mathrm{Te}_{3}$ nanoplate. From the SAED pattern, it can be concluded that the top/bottom surfaces are both $\{0001\}$ facets, whereas the six side surfaces are all $\{11 \overline{2} 0\}$ facets. From the lattice fringe image, it is observed that the lattice spacing of the hexagonal lattice fringes is $0.217 \mathrm{~nm}$, which is in good agreement with previous values for the interplaner lattice spacing of $\{11 \overline{2} 0\}$ planes. $^{7,8}$ The continuous lattice fringes oriented in the same direction in Figure 1(f) and the sharp diffraction pattern in Figure 1(e) both indicate that the $\mathrm{Bi}_{2} \mathrm{Te}_{3}$ nanoplates are single crystal and essentially defect-free.

To study the growth mechanism and morphology of these 2D nanostructures, the growth conditions were varied, including carrier gas flow rate and growth time. Figures 2(a)-2(c) show representative SEM images of $\mathrm{Bi}_{2} \mathrm{Te}_{3}$ nanostructures grown for a fixed growth time of $1 \mathrm{~h}$, but with different Ar carrier gas flow rates $(60,80$, and $130 \mathrm{sccm}$ for samples B, C, and D, respectively). It is observed that hexagonal $\mathrm{Bi}_{2} \mathrm{Te}_{3}$ nanoplates are formed in all these samples, but with different average dimensions of approximately 4.5, 7.1, and $7.6 \mu \mathrm{m}$ for samples $\mathrm{B}, \mathrm{C}$, and $\mathrm{D}$, respectively. When combined with the result from sample $\mathrm{A}$, it is evident that the lateral dimension of $\mathrm{Bi}_{2} \mathrm{Te}_{3}$ nanoplates increases with increasing Ar carrier gas flow rate up to an Ar carrier gas flow rate of $100 \mathrm{sccm}$, and then decreases with a further increase in the Ar flow rate, which are statistically summarized in Figure 2(d). The increase of nanoplate lateral dimension can be attributed to an increasing amount of $\mathrm{Bi}_{2} \mathrm{Te}_{3}$ precursor vapour transported onto the substrates as the $\mathrm{Ar}$ carrier gas flow rate is increased. ${ }^{15}$ Upon reaching the substrate, the $\mathrm{Bi}_{2} \mathrm{Te}_{3}$ precursor molecules decompose into $\mathrm{Bi}$ and Te atoms, which condense on appropriate sites to nucleate and grow on the substrate surface. Thus, for a fixed growth time, higher Ar carrier gas flow rate provides more $\mathrm{Bi}$ and $\mathrm{Te}$ atoms on the substrate surface and leads to a higher growth rate and larger nanoplates. However, the observed decrease in lateral dimension of the $\mathrm{Bi}_{2} \mathrm{Te}_{3}$ nanoplates with a further increase in the carrier gas flow rate above $100 \mathrm{sccm}$ indicates that the nanoplate growth is a consequence of two competing processes. At low carrier gas flow rates, carrier gas-related vapour transport plays the dominant role in material growth, whereas at high carrier gas flow rates, some other competing mechanism plays a significant role in material growth. The detailed growth mechanism of these $\mathrm{Bi}_{2} \mathrm{Te}_{3}$ nanoplates will be discussed later in this work. Note that no significant change is observed in the thickness of the $\mathrm{Bi}_{2} \mathrm{Te}_{3}$ nanoplates with increasing carrier gas flow rate, primarily due to the much lower growth rate along the $\langle 0001\rangle$ direction in comparison to the $\langle 11 \overline{2} 0\rangle$ direction.

Apart from the carrier gas flow rate, the growth time has also been varied to study its effect on the morphology of the $\mathrm{Bi}_{2} \mathrm{Te}_{3}$ nanostructures. Figure 3 shows representative SEM images of the $\mathrm{Bi}_{2} \mathrm{Te}_{3}$ nanostructures grown with a fixed $\mathrm{Ar}$ 

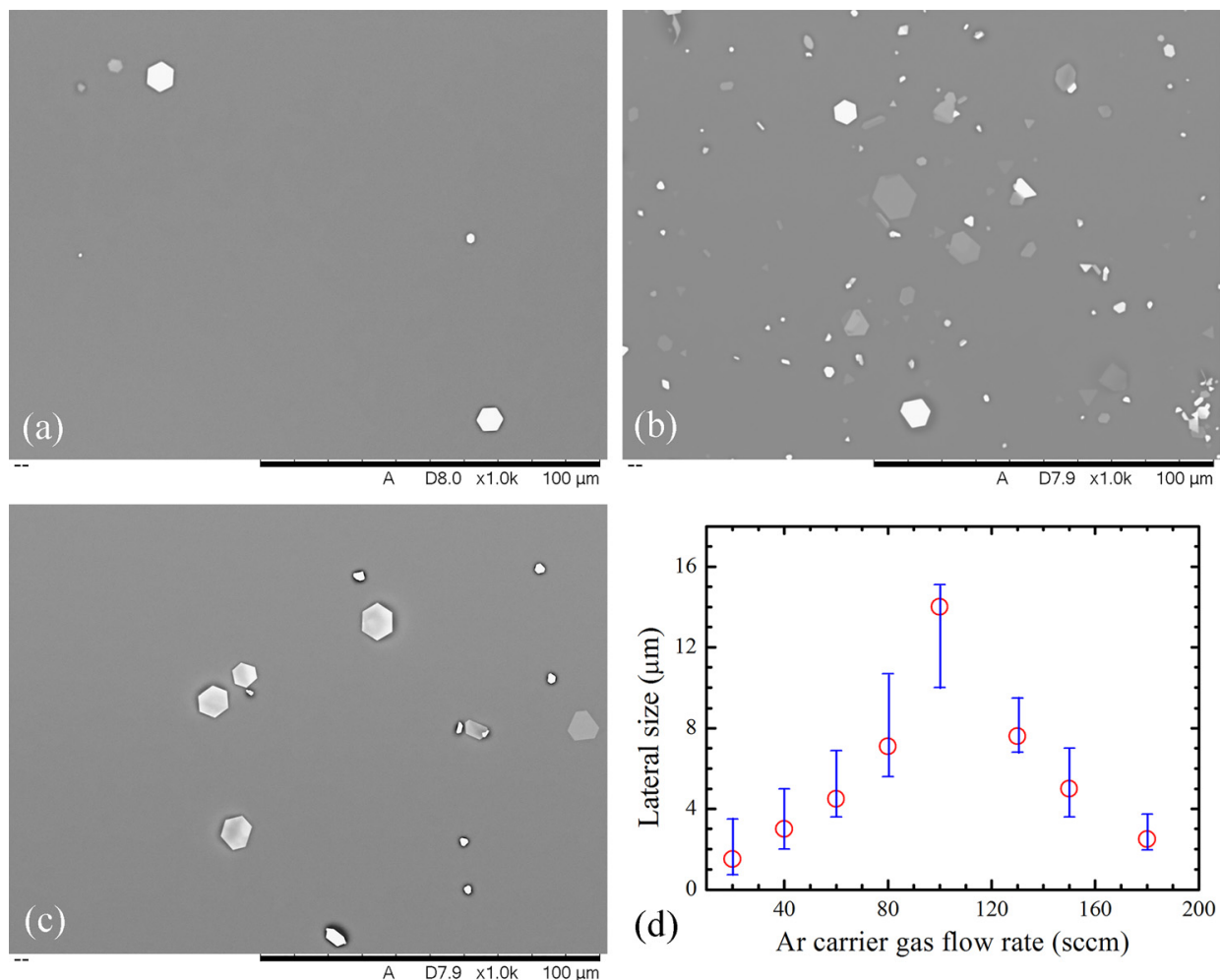

FIG. 2. Representative SEM images of (a) samples B, (b) sample C, and (c) sample D, and (d) statistical summary of $\mathrm{Bi}_{2} \mathrm{Te}_{3}$ nanoplate's lateral size and its distribution in samples A, B, C, and D. Note in (d) the data of samples grown with a growth time of $1 \mathrm{~h}$ and a Ar carrier gas flow rate of 20,40, 150, and $180 \mathrm{sccm}$ are also provided for the readers' information. carrier gas flow rate of $100 \mathrm{sccm}$, but for different growth times: $10 \mathrm{~min}, 30 \mathrm{~min}$, and $2 \mathrm{~h}$ for samples $\mathrm{E}, \mathrm{F}$, and $\mathrm{G}$, respectively. Although the $\mathrm{Bi}_{2} \mathrm{Te}_{3}$ nanostructures also present hexagonal shapes for samples E, F, and G, the average lateral dimension was found to vary significantly with increasing growth time, giving dimensions of approximately $0.76,6.4$, and $19.5 \mu \mathrm{m}$ for samples $\mathrm{E}, \mathrm{F}$, and $\mathrm{G}$, respectively. When combined with sample A, it is evident that the lateral size of $\mathrm{Bi}_{2} \mathrm{Te}_{3}$ nanoplates increases with increasing growth time, which is as expected from the kinetic characteristics of $\mathrm{Bi}_{2} \mathrm{Te}_{3}$ nanoplate growth. That is, with increasing growth time, the $\mathrm{Bi}$ and $\mathrm{Te}$ atoms on the substrate surface will continue to migrate and diffuse to find the most energetically favourable nucleation site to attach to and grow, which are evidently the edges of existing nanoplates. This leads to the formation of nanoplates with larger lateral size with increasing growth time, as observed in Figure 3, which indicates that the lateral growth of $\mathrm{Bi}_{2} \mathrm{Te}_{3}$ nanoplates found in this work follows the same fundamental kinetic principles of epitaxial growth of materials, which has been widely observed via MBE and MOCVD. ${ }^{16-18}$

Based on the above discussion, a model is proposed to describe and explain the growth mechanism of $\mathrm{Bi}_{2} \mathrm{Te}_{3}$ nanoplates, which is schematically demonstrated in Figure 4. As the growth of $\mathrm{Bi}_{2} \mathrm{Te}_{3}$ nanoplates is still a vapour phase deposition process, the model proposed is based on the fundamental principles of chemical vapour deposition. Figure 4(a) depicts the processes involved in the growth of $\mathrm{Bi}_{2} \mathrm{Te}_{3}$ nanostructures, in which it is assumed that the boundary layer due to steady state gas flow is stagnant. The $\mathrm{Bi}_{2} \mathrm{Te}_{3}$ precursor molecules initially (1) diffuse through the boundary layer and reach the substrate surface, then (2) are adsorbed on the substrate surface, then (3) decompose to form active Bi and Te atoms, and then (4) diffuse along the substrate surface, and nucleate and grow on energetically preferential sites.
Any unreacted atoms/molecules then (5) are desorbed from the surface, and then (6) diffuse from the surface through the boundary layer and are eventually swept away by the bulk gas flow. ${ }^{15}$ Depending upon whether the processes take place in the boundary layer (1 and 6 in Figure 4(a)) or close to the surface (2-4 in Figure 4(a)), they can be categorized into two regimes: the mass transport regime, primarily involving diffusion through the boundary layer, and the surface reaction regime. Therefore, there are two fluxes of the active molecules/atoms that coexist on the surface: a flux of active molecules/atoms that diffuse from the boundary layer$F_{\text {mass-transport }}$, and another flux associated with the rate at which the active molecules/atoms are consumed at the substrate surface to grow $\mathrm{Bi}_{2} \mathrm{Te}_{3}-F_{\text {surface-reaction. The }}$ two fluxes are in series, and the slower of the two processes is the rate-limiting step for material growth. At the steady state, $F_{\text {mass-transport }}=F_{\text {surface-reaction }}$. Based on this model, there are three regimes: $F_{\text {mass-transport }} \gg F_{\text {surface-reaction }}$ (surface reaction limited (SRL) regime), $F_{\text {mass-transport }} \sim F_{\text {surface-reaction }}$ (mixed regime), and $F_{\text {mass-transport }} \ll F_{\text {surface-reaction }}$ (mass transport limited (MTL) regime). ${ }^{15}$

Generally, for a given surface temperature and fixed growth time, when the carrier gas flow rate is low, mass transport through the boundary layer is the rate limiting step ( $F_{\text {mass-transport }} \ll F_{\text {surface-reaction }}$ ), whereas when the carrier gas flow rate is high, the surface reaction is usually the rate limiting step ( $\left.F_{\text {mass-transport }} \gg F_{\text {surface-reaction }}\right)$, as shown in Figure 4(b). ${ }^{15}$ From the above discussion, it can be speculated that samples $\mathrm{A}, \mathrm{B}$, and $\mathrm{C}$ are in the MTL regime, while sample D is in the SRL regime. In the MTL regime, the diffusion of active molecules through the boundary layer onto the substrate surface is lower than those consumed by reaction/material growth. In this case, the active $\mathrm{Bi}_{2} \mathrm{Te}_{3}$ molecules diffusing through the boundary layer increase with increasing carrier gas flow rate, resulting in an increase of 

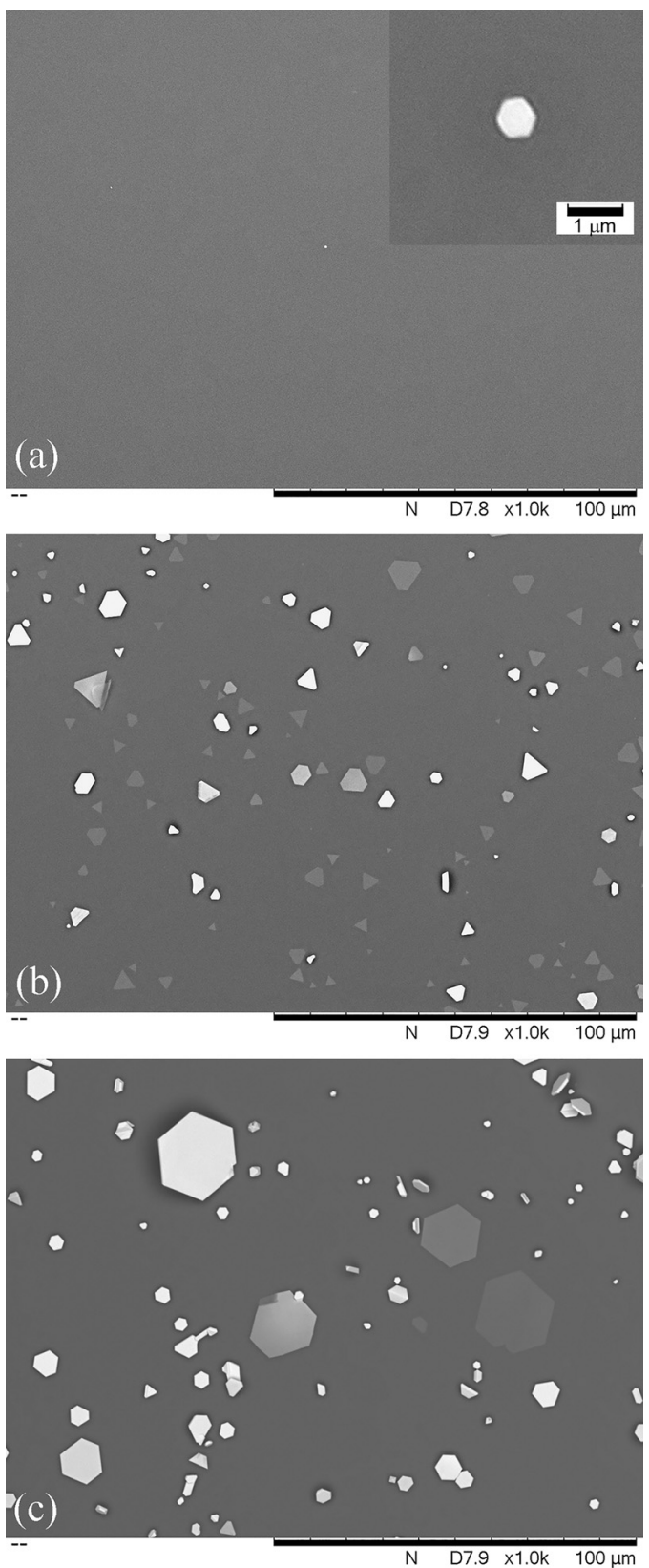

FIG. 3. Representative SEM images of (a) sample E, (b) sample F, and (c) sample G.

decomposed $\mathrm{Bi}$ and $\mathrm{Te}$ atoms on the substrate surface. This results in a higher growth rate and larger nanoplate lateral dimensions with increasing Ar carrier gas flow rate, as observed for samples A, B, and C. However, when the carrier gas flow rate increases to a very high flow rate $(\sim 130$ sccm in this work), the system enters the SRL regime, in which the active $\mathrm{Bi}_{2} \mathrm{Te}_{3}$ molecules diffusing through the boundary layer onto the substrate surface is greater than those consumed by reaction/material growth. In this case, theoretically, the material growth rate will saturate if the carrier gas flow rate is increased further, which should result in $\mathrm{Bi}_{2} \mathrm{Te}_{3}$ nanoplates of similar size to those observed in sample D. However, due to heat loss via convection, a high carrier gas flow rate will eventually result in a temperature decrease of the substrate even the nominal growth temperature was kept constant throughout the study. Note the nominal growth temperature is monitored by a thermocouple

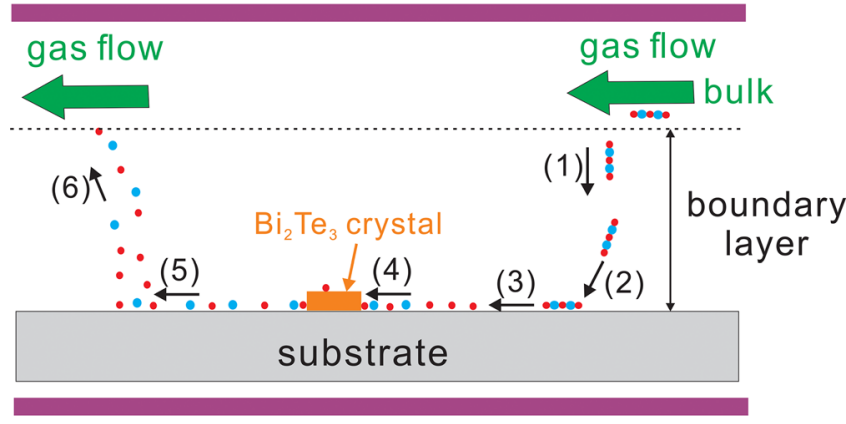

(a)

furnace tube

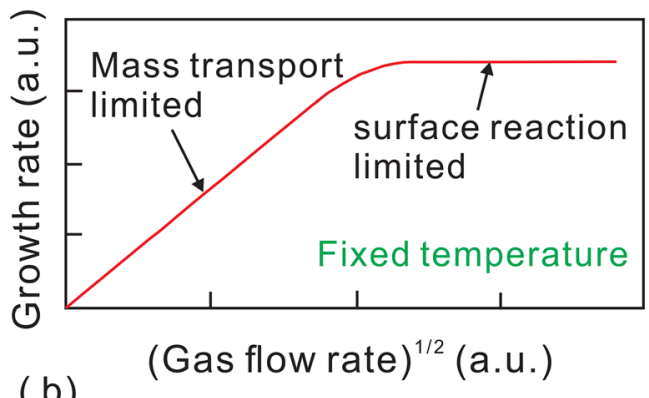

FIG. 4. Schematic growth model for phase deposition synthesis of $\mathrm{Bi}_{2} \mathrm{Te}_{3}$ nanoplates: (a) processes involved during the phase deposition synthesis of $\mathrm{Bi}_{2} \mathrm{Te}_{3}$ nanoplates; and (b) influence of gas flow rate on the growth rate of $\mathrm{Bi}_{2} \mathrm{Te}_{3}$ nanoplates.

placed outside the quartz reaction tube, and thus not necessarily the true temperature of the substrate surface. Importantly, $F_{\text {surface-reaction }}$ is proportional to surface reaction constant $K_{s}$, which is very sensitive to temperature $\left(K_{s} \propto \exp \left(-\frac{E_{a}}{k T}\right)\right.$, where $E_{a}, k$, and $T$ are the apparent activation energy of the growth, Boltzmann's constant, and surface temperature, respectively). ${ }^{15}$ Thus, $F_{\text {surface-reaction }}$ will decrease rapidly with reducing surface reaction temperature. This leads to a decreased growth rate and smaller lateral dimension for $\mathrm{Bi}_{2} \mathrm{Te}_{3}$ nanoplates in sample $\mathrm{D}$ in comparison to those in sample $\mathrm{C}$, as observed in Figure 2.

For samples E, F, and G, the system is in the MTL regime, since the carrier gas flow rate is kept constant at 100 sccm. With increasing growth time, the $\mathrm{Bi}$ and $\mathrm{Te}$ atoms have sufficient time to diffuse and migrate on the substrate surface, and thus find the most energetically favourable sites to attach and grow, which are usually the edges of the existing $\mathrm{Bi}_{2} \mathrm{Te}_{3}$ nanoplates. This leads to the formation of nanoplates with larger lateral dimension with increasing growth time, as observed in Figure 3.

The formation of a plate geometry indicates a very large difference between the material growth rate along the $\langle 0001\rangle$ direction compared with that along the $\langle 11 \overline{2} 0\rangle$ direction. Since $\mathrm{SiO}_{2}$ is amorphous, the initial nucleation of $\mathrm{Bi}_{2} \mathrm{Te}_{3}$ on the $\mathrm{SiO}_{2}$ surface of the substrate takes place randomly, and the nuclei have the c crystal axis perpendicular to the substrate surface. Due to the layered structure of $\mathrm{Bi}_{2} \mathrm{Te}_{3}$ crystals, atoms at the edge of the nuclei have dangling bonds ready to bind covalently with incoming atoms, whereas the top 
surface is terminated with chemically saturated Te atoms. ${ }^{8}$ Thus, material growth proceeds via a highly anisotropic process, since atoms adsorbed onto the top surface from the gas phase cannot form covalent bonds with the top Te atoms and, consequently, tend to diffuse to the edges to attach. Therefore, the nanoplate lateral dimension grows much faster than the vertical dimension, which is confirmed by the large ratio $(>1270)$ of lateral/vertical dimensions observed in Figure 1.

In conclusion, the influence of growth conditions on the morphology of $2 \mathrm{D} \mathrm{Bi}_{2} \mathrm{Te}_{3}$ nanoplates has been investigated in detail, leading to the development of a growth model that describes the vapour phase growth of these nanoplates. The formation of plate geometry is a consequence of the large bonding anisotropy of $\mathrm{Bi}_{2} \mathrm{Te}_{3}$ materials, and is dominated by the diffusion of $\mathrm{Bi}$ and $\mathrm{Te}$ atoms on the substrate surface. Under low carrier gas flow rates $(60 \mathrm{sccm}-100 \mathrm{sccm})$, growth is mass-transport limited, which causes the lateral size of $\mathrm{Bi}_{2} \mathrm{Te}_{3}$ nanoplates to increase with increasing $\mathrm{Ar}$ carrier gas flow rate. Under high carrier gas flow rates $(>100 \mathrm{sccm})$, growth is surface-reaction limited, which causes the nanoplate lateral size to decrease with increasing Ar carrier gas flow rate due to a decrease in substrate temperature. As expected, the nanoplate lateral size was found to increase with increasing growth time as a result of the kinetic feature of vapour phase growth. The simple growth model discussed in this paper provides an effective guide to achieving controlled growth of $\mathrm{Bi}_{2} \mathrm{Te}_{3}$ nanostructures, which can also be applied to other 2D material systems.

This work was supported by the Australian Research Council (FT130101708), Universities Australia-DAAD German Research cooperation scheme (2014-2015), and a
Research Collaboration Awards from The University of Western Australia. Facilities used in this work are supported by the WA node of Australian National Fabrication Facility (ANFF).

${ }^{1}$ H. J. Zhang, C. X. Liu, X. L. Qi, X. Dai, Z. Fang, and S. C. Zhang, Nat. Phys. 5, 438 (2009).

${ }^{2}$ M. Z. Hasan and C. L. Kane, Rev. Mod. Phys. 82, 3045 (2010).

${ }^{3}$ D. Teweldebrhan, V. Goyal, and A. A. Balandin, Nano Lett. 10, 1209 (2010).

${ }^{4}$ L. D. Alegria, N. Yao, and J. R. Petta, Phys. Status Solidi RRL 8, 991 (2014).

${ }^{5}$ Y. Wang, F. Xiu, L. Cheng, L. He, M. Lang, J. Tang, X. Kou, X. Yu, X. Jiang, Z. Chen, J. Zou, and K. L. Wang, Nano Lett. 12, 1170 (2012).

${ }^{6}$ D. Kong, J. C. Randel, H. Peng, J. J. Cha, S. Meister, K. Lai, Y. Chen, Z. Shen, H. C. Manoharan, and Y. Cui, Nano Lett. 10, 329 (2010).

${ }^{7}$ H. Li, J. Cao, W. Zheng, Y. Chen, D. Wu, W. Dang, K. Wang, H. Peng, and Z. Liu, J. Am. Chem. Soc. 134, 6132 (2012).

${ }^{8}$ D. Kong, W. Dang, J. J. Cha, H. Li, S. Meister, H. Peng, Z. Liu, and Yi. Cui, Nano Lett. 10, 2245 (2010).

${ }^{9}$ R. B. Jacobs-Gedrim, C. A. Durcan, N. Jain, and B. Yu, Appl. Phys. Lett. 101, 143103 (2012)

${ }^{10}$ G. Hao, X. Qi, Y. Liu, Z. Huang, H. Li, K. Huang, J. Li, L. Yang, and J. Zhong, J. Appl. Phys. 111, 114312 (2012).

${ }^{11}$ L. Guo, B. C. Ivey, A. Aglan, C. Tang, J. Song, C. H. Turner, R. M. Frazier, A. Gupta, and H. T. Wang, ECS Solid State Lett. 2, 19 (2013).

${ }^{12}$ Y. Zhao, M. de la Mata, R. L. J. Qiu, J. Zhang, X. Wen, C. Magen, X. P. A. Gao, J. Arbiol, and Q. Xiong, Nano Res. 7, 1243 (2014).

${ }^{13}$ Y. Jiang, X. Zhang, Y. Wang, N. Wang, D. West, S. Zhang, and Z. Zhang, Nano Lett. 15, 3147 (2015).

${ }^{14}$ K. M. F. Shahil, M. Z. Hossain, V. Goyal, and A. A. Balandind, J. Appl. Phys. 111, 054305 (2012).

${ }^{15}$ J. D. Plummer, M. Deal, and P. D. Griffin, "Chapter 9: Thin film deposition" in Silicon VLSI Technology: Fundamentals, Practice, and Modeling, 1st ed. (Prentice Hall, Upper Saddle River, NJ, 2000).

${ }^{16}$ W. Lei, H. H. Tan, and C. Jagadish, Appl. Phys. Lett 95, 013108 (2009).

${ }^{17}$ C. Zhao, Y. H. Chen, C. X. Cui, B. Xu, J. Sun, W. Lei, L. K. Lu, and Z. G. Wan, J. Chem. Phys. 123, 094708 (2005).

${ }^{18}$ L. K. Yu, B. Xu, Z. G. Wang, P. Jin, C. Zhao, W. Lei, J. Sun, K. Li, L. J. $\mathrm{Hu}$, and L. Y. Liang, J. Cryst. Growth 282, 173 (2005). 